\title{
Evaluation of BPPV with vertebral artery values
}

This article was published in the following Dove Press journal:

Neuropsychiatric Disease and Treatment

\author{
Alper Yazıcı' \\ Yusuf İnanç² \\ 'Otorhinolaryngology Department. \\ University of Gaziantep Medical \\ Faculty, Gaziantep, Turkey; ${ }^{2}$ Neurology \\ Department, University of Gaziantep \\ Medical Faculty, Gaziantep, Turkey
}

Objectives: The aim of this study was to investigate the association between benign paroxysmal positional vertigo (BPPV) and vertebral artery (VA) flow rates and diameters by using Doppler ultrasonography in patients with BPPV. Additionally, we aimed to investigate the abnormalities of VA values obtained by extracranial color-coded duplex sonography in BPPV, which could be accepted as a precursor lesion for posterior cerebral ischemic stroke.

Materials and methods: Between September 2017 to February 2018, 50 patients diagnosed with BPPV were enrolled in this study. Otorhinolaryngology medical examination and medical history assessment were performed at the otorhinolaryngology clinic. Afterward, neurologic examination and ultrasonography of VAs was undertaken in the neurology clinic of University of Gaziantep Medical Faculty.

Results: The types of BPPV found were as follows: right posterior in 27 (54\%), right lateral in $4(8 \%)$, left posterior in $16(32 \%)$, and left lateral in $3(6 \%)$, respectively. The most common variables found in the BPPV were smoking (26\%), hypertension (26\%), and hyperlipidemia (22\%). Conclusion: VA flow rates and diameters were found to be affected at the site of BPPV. These findings might suggest that BPPV could be related to an atherosclerotic milieu and may be a precursor clinical condition for future intracranial atherosclerotic diseases.

Keywords: benign paroxysmal positional vertigo, vertebral artery, Doppler ultrasonography, atherosclerotic disease

\section{Introduction}

Vertigo is a complaint which neurology; ear, nose, and throat; and emergency medicine practitioners deal with in their daily practice. ${ }^{1,2}$ Benign paroxysmal positional vertigo (BPPV) is the most common type of vertigo. ${ }^{3} \mathrm{BPPV}$ is generally accepted as a benign clinical condition which has been reported in 2 different studies as having a prevalence of 10.7 and 60.4 cases per 100,000 populations, respectively. Additionally, BPPV is one of the most common etiologies of vertigo and balance disorders among the elderly. ${ }^{4}$ The etiology of BPPV remains unclear. Head trauma, infection, and middle ear surgery are the most prevalent risk factors which predispose individuals to BPPV. ${ }^{5}$ The pathophysiological mechanism of BPPV is believed to be the displacement of calcium deposits to the semicircular canals that could result in activation of the afferent vestibular nerve fibers. ${ }^{6,7}$ The most affected semicircular canal is the posterior one, and this is responsible for $90 \%$ of BPPV. ${ }^{3}$

Vertebral artery (VA) is the most affected vascular structure at the posterior circulation abnormalities. ${ }^{8}$ The basilar artery formed by the merging of VAs is responsible for the vascular supply of the 4 vestibular nuclei which are located at the base of the fourth ventricle. Additionally, vestibular structures of the internal ear are supplied by the labyrinthine artery, which is a branch of VA.

Extracranial color-coded duplex sonography (ECCSVA) of VAs can be a useful screening tool for the evaluation of posterior cerebral circulation. ${ }^{9}$ ECCSVA is a useful 
imaging modality which is cost-effective and can be easily performed at the clinic.

The purpose of this study was to investigate the association between BPPV and VA flow rates and diameters by using Doppler ultrasonography in patients with BPPV. Additionally, we aimed to investigate the abnormalities of VA values obtained by ECCSVA in BPPV, which could be accepted as a precursor lesion for posterior cerebral ischemic stroke.

\section{Materials and methods Subjects}

From September 2017 to February 2018, 50 patients, (10 males/40 females) (between the ages of 19 and 84 years) diagnosed with BPPV were enrolled in this prospective study. Physical examination data and histories of the patients were obtained from otorhinolaryngology clinic, and ECCSVA was performed in the neurology clinic of University of Gaziantep Medical Faculty. The history regarding hypertension, myocardial infarction, cerebrovascular incidents, diabetes mellitus, thyroid dysfunction, and smoking data were obtained from all of the patients and also rechecked from the medical record system. All patients underwent detailed ear, throat, and nose examination and a detailed neurological examination. Participants who were under the age of 18 and/or had central vertigo, ear drainage, or any disability to prevent the performance of a vestibular exam and those who had a history of cerebrovascular event or transient ischemic attack were excluded from this study. Head thrust, rotating chair, and Dix-Hallpike tests were applied to all patients who had a complaint of vertigo which lasted 1-2 minutes. Also, barbecue roll test was administered to the patients who were suspected of having posterior semicircular canal positional vertigo.

\section{Radiologic evaluation}

BPPV-diagnosed patients were assessed with extracranial color-coded duplex sonography LOGIQ P5 portable ultrasound equipment (GE Healthcare, Buckinghamshire, UK) at 5-12 MHz scanning frequency. The measurements were performed between the segments of VA between the C4 and C6 vertebral bodies. The parameters of VA diameter (VAD), peak systolic index (PSI), pulsatil index (PI), resistive index, and end-diastolic flow velocities were obtained by color-coded duplex sonography examination. The intraarterial diameter was calculated between the intimae layers of VAs, which were assessed in the long vessel section that is perpendicular to the axis. To provide the proper shaft, an approximately $60^{\circ}$ angle between the wall of the vessels and ray beams was created.

\section{Statistics}

One-sample $t$-test used to compare the sides of VA diameter and PSI among the BPPV subtypes. Statistical analyses were conducted using SPSS 22 (IBM Corporation, Armonk, NY, USA), and $p$-values $<0.05$ were considered statistically significant.

\section{Ethics statement}

The approval for this study was obtained from the ethical committee of the University of Gaziantep. Written informed consent was received from all individuals who participated in this study.

\section{Results}

A total of 50 patients were included in this study. The study population was composed of 40 females $(80 \%)$ and 10 males (20\%). The age of the research group ranged between 19 and 83 years. The mean age was $44.08 \pm 16.5$. Atherosclerotic risk factors in BPPV patients are shown in Table 1. The most common atherosclerotic risk factors were hypertension, smoking (26\%), and hyperlipidemia (22\%). Types of BPPV were found to be distributed as right posterior in 27 (54\%), right lateral in $4(8 \%)$, left posterior in $16(32 \%)$, and left lateral in $3(6 \%)$.

Looking into VA patterns, the VA having bigger diameter was dominant; from this standpoint, the left VA dominance was seen in 29 patients, whereas 18 patients showed right VA dominance, and no supremacy was detected in 3 patients. Fifty percent of males had right VA dominance, while among females $55 \%$ had left VA dominance. VAD values were found to be statistically significant between 2 genders $(p<0.05)$. The diameter of VA below $2.6 \mathrm{~mm}$ and/or PSI below $35 \mathrm{~mL} / \mathrm{min}$ was defined as hypoplasia. Lack of any flow in VA was defined high-grade stenosis.

Patients with the hypoplasia of left and right VA numbered $30(60 \%)$ and $22(44 \%)$, respectively. High-grade stenosis was displayed in 2 left VAs and was in 1 right VA.

In-depth analysis of VA flow variables revealed the following: the diameter of left VA was found to be an

Table I Atherosclerotic risk factors of BPPV patients

\begin{tabular}{ll}
\hline $\begin{array}{l}\text { Atherosclerotic } \\
\text { risk factors }\end{array}$ & Percentage \\
\hline Hypertension & $13(26 \%)$ \\
Hyperlipidemia & $1 \mathrm{I}(22 \%)$ \\
Smoking & $13(26 \%)$ \\
Diabetes mellitus & $10(20 \%)$ \\
Thyroid dysfunction & $10(20 \%)^{\mathrm{a}}$ \\
\hline
\end{tabular}

Note: ${ }^{2}$ Thyroid dysfunction in 10 patients includes 3 with hyperthyroidism and 7 with hypothyroidism.

Abbreviation: BPPV, benign paroxysmal positional vertigo. 
Table 2 Overall values of VA in BPPV patients

\begin{tabular}{llll}
\hline Parameter & Mean \pm SD & Minimum & Maximum \\
\hline LAD & $0.39 \pm 0.11$ & 0.19 & 0.84 \\
LPSI & $32.8 I \pm 10.96$ & 0 & 60 \\
LPI & $2.32 \pm 1.6$ & 0 & 7.5 \\
LRI & $0.87 \pm 0.3$ & 0 & 2.1 \\
LEDI & $6.12 \pm 7.68$ & 0 & 22 \\
RAD & $0.37 \pm 0.09$ & 0.2 & 0.77 \\
RPSI & $36.3 \pm 14.32$ & 0 & 73 \\
RPI & $2.16 \pm 1.38$ & 0 & 7.7 \\
RRI & $I \pm 0.61$ & 0 & 5 \\
REDI & $6.29 \pm 8.05$ & 0 & 7.7 \\
\hline
\end{tabular}

Abbreviations: BPPV, benign paroxysmal positional vertigo; LAD, left vertebral artery diameter; LEDI, left end-diastolic index; LPI, left vertebral artery pulsality index; LPSI, left vertebral artery peak systolic index; LRI, left resesitive index; RAD, right vertebral artery diameter; REDI, right end-diastolic index; RPI, right vertebral artery pulsality index; RPSI, right vertebral artery peak systolic index; RRI, right resesitive index; VA, vertebral artery.

average of $3.9+0.11 \mathrm{~mm}$, the mean PSI was found to be $36.3+14.32$ in the right and $32.81+10.96$ in the left, the mean resistive index detected in the right was $1+0.61$ and it was $0.87+0.30$ in the left $\mathrm{VA}$, the averages of values were $2.16+1.38$ and $2.16+1.38$ in the left and right, respectively, end-diastolic velocity was found to be $6.29+8.05$ in the right and $6.12+7.68$ in the left (Table 2). Also, the values of the VA in each gender, which were calculated by ECCSVA, are shown in Table 3.

Vertigo is divided into 4 subtypes based on the involvement of the semicircular canals. In this study, patients with left posterior BPPV showed a measurement of left and right VAD of $0.40 \pm 0.13 \mathrm{~cm}$ and $0.41 \pm 0.13 \mathrm{~cm}$, respectively. The left and right VAD were calculated to be $0.31 \pm 0.1 \mathrm{~cm}$ and $0.35 \pm 0.05 \mathrm{~cm}$ in left lateral BPPV patients. Additionally, patients suffering from right posterior BPPV had a left VAD of $0.39 \pm 0.1 \mathrm{~cm}$ and $0.34 \pm 0.06 \mathrm{~cm}$ for the right one.

Table 3 Comparison of the BPPV on different sides based on VA variables

\begin{tabular}{llll}
\hline Parameter & Left BPPV & Right BPPV & P-value \\
\hline LAD & $0.38 \pm 0.13$ & $0.39 \pm 0.09$ & 0.884 \\
LPSI & $27.71 \pm 11.74$ & $36.13 \pm 9.43$ & 0.008 \\
LPI & $2.83 \pm 2$ & $2.03 \pm 1.25$ & 0.092 \\
LRI & $0.82 \pm 0.44$ & $0.9 \pm 0.18$ & 0.405 \\
LEDI & $4.92 \pm 7.3$ & $6.75 \pm 8.05$ & 0.428 \\
RAD & $0.4 \pm 0.12$ & $0.34 \pm 0.06$ & 0.028 \\
RPSI & $38.86 \pm 13.76$ & $34.55 \pm 14.87$ & 0.314 \\
RPI & $2.39 \pm 1.51$ & $1.99 \pm 1.31$ & 0.331 \\
REDI & $7.46 \pm 9.05$ & $5.1 \pm 7.06$ & 0.311 \\
\hline
\end{tabular}

Note: Data are presented as mean \pm standard deviation.

Abbreviations: BPPV, benign paroxysmal positional vertigo; LAD, left vertebral artery diameter; LEDI, left end-diastolic index; LPI, left vertebral artery pulsality index; LPSI, left vertebral artery peak systolic index; LRI, left resesitive index; RAD, right vertebral artery diameter; REDI, right end-diastolic index; RPI, right vertebral artery pulsality index; RPSI, right vertebral artery peak systolic index; RRI, right resesitive index; VA, vertebral artery.
Left and right $V A D$ were found to have sizes of $0.4 \pm 0.03 \mathrm{~cm}$ and BPPV $0.38 \pm 0.04 \mathrm{~cm}$ in right lateral BPPV patients, respectively.

Left lateral BPPV and left posterior BPPV variables were combined to form the left-type BPPV group. The right-type BPPV group was assessed in the same manner. The VA variable comparison based on left or right BPPV is displayed in Table 3. According to Student's $t$-test, a comparison of VA diameter and the VA PSI values were found to be statistically significant.

\section{Discussion}

To the best of our knowledge, with a broad review of the literature, this study seems to be the first to determine the VA parameters by using ECCSVA in BPPV patients. In this study, we have shown that the VAD and the VA blood flow rates, which were measured by Doppler ultrasonography (ECCSVA), have lower values on the side of BPPV.

Previous studies displayed that the atherosclerotic risk factors such as hypertension, hyperlipidemia, smoking, migraine, and diabetes exist with high percentages in BPPV patients. ${ }^{4,10}$ These atherosclerotic risk factors were found in nearly 1 of 3 BPPV patients in our study. Furthermore, the association of atherosclerosis with not only BPPV but also with sudden hearing loss was reported. ${ }^{11,12}$ However, studies regarding the relation of atherosclerosis either with vestibular neuritis or Meniere disease are scarce. One of the explanations for the linkage between these risk factors and BPPV is that atherosclerosis of VA may trigger the detachment of otoconia from the otolith membrane by means of labyrinthine injury caused by oxidative radicals that are produced during ischemia. ${ }^{13}$ From this standpoint, it could be argued that the other parts of vestibulocochlear system might be affected; however, we focused on BPPV in this study; therefore, we did not perform further investigations. One of the explanations for the linkage between these risk factors and BPPV was that atherosclerosis of VA could initially cause labyrinthine ischemia, which then facilitates detachment of otoconia from the otolith membrane. ${ }^{13}$

In addition to the atherosclerotic risk factors, the anatomical integrity of VAs, which are responsible for posterior cerebral perfusion, is closely related to vertigo and stroke. Association between VA hypoplasia and posterior ischemic cerebral stroke has been consistently highlighted in previous studies. ${ }^{14,15}$ Hypoplasia of the VA has not been accepted as an absolute independent risk factor for stroke, but especially the hypoplasia of VA along with the other risk factors, especially hypertension, smoking, and diabetes, is more likely to lead to development of an ischemic cerebrovascular event. ${ }^{16}$ 
Alternatively, recent studies also pointed that there was an increased risk for ischemic stroke in BPPV patients. ${ }^{10}$ According to Kao et al, ${ }^{10}$ the stroke percentages were found to be $4.5 \%$ in BPPV patients after 9-year follow-up. Also, Tan et $\mathrm{al}^{5}$ found that nearly $25 \%$ of BPPV patients who underwent magnetic resonance imaging exhibited asymptomatic infarcts and microhemorrhage. However, these 2 articles did not mention the flow and hypoplasia rates of the VA, which could be accepted as a risk factor for the posterior cerebral stroke despite the fact that detailed information was collected after a long follow-up. ${ }^{15}$

In our study, most of the patients exhibited a decrease in the posterior cerebral blood flow on the side where BPPV was diagnosed. Also, hypoplasia of VA has been demonstrated in nearly $50 \%$ of BPPV patients. Additionally, Zhang et $\mathrm{al}^{17}$ had shown abnormality in cerebral circulation of BPPV patients ranging at $57.1 \%$, and they also found that the flow rate in posterior cerebral arteries was higher in BPPV patients compared to the control group. The values of this study were collected from the assessment of posterior cerebral flow of the pediatric population diagnosed with BPPV. ${ }^{17}$ In contrast, we found the posterior cerebral arteries flow rates decreased on the side of BPPV. This result may suggest that the flow rates in the BPPV sides decreased with time owing to the influence of atherosclerotic risk factors.

Angiography is the gold-standard technique to enable the visualization of posterior cerebral arteries with an active blood flow evaluation. ${ }^{18}$ Other noninvasive advanced diagnostic techniques for VA imaging are angio-computed tomography and contrast-enhanced magnetic resonance angiography, known to have higher accuracy to assess vascular abnormalities especially for posterior cerebral arteries. ${ }^{18}$ Nevertheless, contrast-enhanced magnetic resonance angiography and angio-computed tomography techniques, which are generally available in the referred center for stroke, are dependent on having an experienced neuroradiologist, and these imaging modalities are more expensive than Doppler ultrasonography. ${ }^{18}$ In the literature, the diagnostic value of Doppler ultrasonography has been investigated by the comparison of different imaging modalities. According to Nazerian et al, ${ }^{9}$ any abnormalities in the VA could be detected by ECCSVA with a sensitivity at 54\% and a specificity at $95 \%$. In the meta-analysis by Khan et al, ${ }^{18}$ the sensitivity and specificity of ECCSVA for diagnosis of vertebral stenosis were $70 \%$ and $98 \%$, respectively, compared with angiography.

One of the limitations of our study was our incapability to evaluate these patients with a magnetic resonance angiography imaging modality, which is more sensitive than ECCSVA. Another weakness was the lack of a control group without any risk factors.

\section{Conclusion}

In conclusion, this prospective study demonstrates that VA blood flow rates and diameters are affected on the side of BPPV. These findings might suggest that BPPV could be related to an atherosclerotic milieu and may be a precursor clinical condition for future intracranial atherosclerotic diseases.

\section{Disclosure}

The authors report no conflicts of interest in this work.

\section{References}

1. Harun A, Agrawal Y. Theuse of fall risk increasing drugs (FRIDs) in patients with dizziness presenting to a neurotology clinic. Otol Neurotol. 2015;36(5):862-864.

2. National Center for Health Statistics. National Hospital Ambulatory Medical Care Survey: 2011 Emergency Department Summary Tables. 2011. Hyattsville, MD: National Center for Health Statistics. Available from: https://www.cdc.gov/nchs/data/ahcd/nhamcs_emergency/2011_ ed_web_tables.pdf. Accessed February 17, 2018.

3. Kim JS, Zee DS. Benign paroxysmal positional vertigo. $N$ Engl J Med. 2014;370(12):1138-1147.

4. von Brevern M, Radtke A, Lezius F, et al. Epidemiology of benign paroxysmal positional vertigo: a population based study. $J$ Neurol Neurosurg Psychiatry. 2006;78(7):710-715.

5. Tan F, Bartels C, Walsh RMC. Our experience with 500 patients with benign paroxysmal positional vertigo: reexploring aetiology and reevaluating MRI investigation. Auris Nasus Larynx. 2018;45(2):248-253.

6. Schuknecht HF. Cupulolithiasis. Arch Otolaryngol. 1969;90(6): 765-778.

7. Hall SF, Ruby RR, McClure JA. The mechanics of benign paroxysmal vertigo. J Otolaryngol. 1979;8(2):151-158.

8. Rozeman AD, Hund H, Westein M, et al. Duplex ultrasonography for the detection of vertebral artery stenosis. Brain Behav. 2017;7(8): $\mathrm{e} 00750$.

9. Nazerian P, Bigiarini S, Pecci R, et al. Duplex sonography of vertebral arteries for evaluation of patients with acute vertigo. Ultrasound Med Biol. 2017;44(3):584-592.

10. Kao CL, Cheng YY, Leu HB, et al. Increased risk of ischemic stroke in patients with benign paroxysmal positional vertigo: a 9-year follow-up nationwide population study in Taiwan. Front Aging Neurosci. 2014;6:108.

11. Rajati M, Azarpajooh MR, Mouhebati M, et al. Is sudden hearing loss associated with atherosclerosis? Iran J Otorhinolaryngol. 2016; 28(86):189-195.

12. Offergeld C, Schellong S, Schmidt A, Pfaar O, Steigerwald C, Zahnert T. Wertigkeit der farbkodierten Duplexsonografie bei neuro-otologischen Krankheitsbildern. Ultraschall der Medizin. 2008;29(6):627-632. German.

13. Ishiyama A, Jacobson KM, Baloh RW. Migraine and benign positional vertigo. Ann Otol Rhinol Laryngol. 2000;109(4):377-380.

14. Gaigalaite V, Vilimas A, Ozeraitiene V, et al. Association between vertebral artery hypoplasia and posterior circulation stroke. BMC Neurol. 2016;16:118.

15. Katsanos AH, Giannopoulos S. Increased risk for posterior circulation ischaemia in patients with vertebral artery hypoplasia: a systematic review and meta-analysis. Eur Stroke J. 2017;2(2):171-177. 
16. Katsanos AH, Kosmidou M, Kyritsis AP, Giannopoulos S. Is vertebral artery hypoplasia a predisposing factor for posterior circulation cerebral ischemic events? A comprehensive review. Eur Neurol. 2013; 70(1-2):78-83.

17. Zhang D, Fan Z, Han Y, et al. Benign paroxysmal vertigo of childhood: diagnostic value of vestibular test and high stimulus rate auditory brainstem response test. Int J Pediatr Otorhinolaryngol. 2012;76(1): $107-110$.
18. Khan S, Rich P, Clifton A, Markus HS. Noninvasive detection of vertebral artery stenosis: a comparison of contrast-enhanced MR angiography, CT angiography, and ultrasound. Stroke. 2009;40(11):3499-3503.

Neuropsychiatric Disease and Treatment

\section{Publish your work in this journal}

Neuropsychiatric Disease and Treatment is an international, peerreviewed journal of clinical therapeutics and pharmacology focusing on concise rapid reporting of clinical or pre-clinical studies on a range of neuropsychiatric and neurological disorders. This journal is indexed on PubMed Central, the 'PsycINFO' database and CAS, and is the official journal of The International Neuropsychiatric Association (INA). The manuscript management system is completely online and includes a very quick and fair peer-review system, which is all easy to use. Visit http://www.dovepress.com/testimonials.php to read real quotes from published authors.

Submit your manuscript here: http://www.dovepress.com/neuropsychiatric-disease-and-treatment-journal 